transformations from child to adult and from sickness to health require ritual to establish new orientations in the mind.

Childhood experiences of prolonged cancer treatment reverberate into adulthood long after treatment has finished. Adult survivors may remain marginalised from their peers despite support from psycho-social professionals (Larcombe et al,2002; Stam, 2005). Paediatricians and Psychologists largely rely on the research methods of objective science, but their reductive processes may not capture holistic experiences which require an empathic science that can understand and grasp the reality of transformations in social health. An empathic science such as Anthropology tends to be underpinned by triangulation whereby evidence from multiple sources converges and emerges as a single account. This paper based on camp observations as a Paediatrician for over ten years, interviews with staff and campers as adults in Europe and USA, and review of protocols and literature claims that the research approaches of Medical Anthropology can elucidate the experiential processes which underpin the social transformation of seriously ill children attending special holiday camps.

Camp experiences are about erasing borderlines between the ill and the healthy. Borderlines have twin functions that can be explained by the single concept of liminality (Szakolczai, 2007):

'Both (functions) problematize the borderline, the limes: in one case it is ignored, in the other accentuated.'

The borderline for children with life threatening illnesses is between two kingdoms whose distinctions are effectively ignored and erased in camp. The liminal experience of fun camps are part of a recognisable tripartite pattern of separation, transition and reintegration rituals summarised by vanGennep as a rite of passage. It enables children move from the kingdom of the sick to the kingdom of the well.

\section{P403 A FIVE YEAR REVIEW OF 'REALLY SICK INPATIENTS WITH ANOREXIA NERVOSA' AT UNIVERSITY HOSPITAL LIMERICK}

Katie Lynam*, Niall Dunworth, Erica Crothers, Cillian Lineen, Orla Neylon, AnneMarie Murphy, John Twomey, Siobhan Gallagher, Clodagh O'Gorman. University Hospital Limerick, Limerick, Ireland

\subsection{6/archdischild-2019-epa.749}

Background The Junior MARSIPAN (Management of Really SIck Inpatients with Anorexia Nervosa) guideline provides paediatricians with a framework for managing anorexia nervosa in the inpatient setting.

Objectives Our aim was to retrospectively review patients in our catchment area with a confirmed diagnosis of anorexia nervosa who required inpatient hospitalisation for management of their eating disorder. We wanted to compare the care provided at our hospital to the recommendations set out in the guidelines.

Methodology We collected data on demographics, morbidity on presentation, number and length of hospital stays, psychosocial stressors and outcomes to date in our cohort over the 5 year period of 2014-2018.

Results We had a total of 10 'really sick inpatients with anorexia nervosa' at University Hospital Limerick during this period of 5 years. There were 7 females and 3 males. Our patients ranged in age from 10 years to 18 years. The average age of first presentation for our patient cohort was 11.75 years. The longest number of days spent in hospital by a patient in one year was 124 days. Stressors at home were a background feature in $75 \%$ of our patients. There was no associated mortality to date in our cohort. Outcomes to date are mostly good although one patient developed obesity and another is on multiple medications for psychiatric comorbidities.

Conclusion There is certainly a link between a greater severity of illness and comorbidities and longer inpatient hospital stays. These cases demonstrate the complexity and variety of presentations to a regional unit. There are significant challenges, in the Irish setting, in providing care to these patients. Resource limitation represents a significant factor in patient outcomes.

\section{P404 THE CHARACTERISTICS OF COMPLETED SUICIDES AMONG CHILD AND ADOLESCENTS BETWEEN 2002- 2013 IN TURKEY}

Tulin Fidan*, Cinar Yenilmez, Setenay Oner. Eskisehir Osmangazi University Faculty of Medicine, Eskisehir, Turkey

\subsection{6/archdischild-2019-epa.750}

Objective Suicide is a complex human behavior that remains an important mental health problem in Turkey and also in the world. Biological, psychological, social, genetic, environmental, economic, cultural and situational factors are known to interact with each other occur suicide so that preventive mental health is essential to stop this behavior.

Methods In this study, completed suicide statistics about the causes and methods between 2002-2013 years under the age 19 were used, published by the Turkey Statistical Institute (TSI). Analyses were performed using chi-square and log-linear methods in SPSS 21.0 software package.

Results The crude suicide rate in Turkey has been increasing year by year.Completed suicides was higher among females in the years 2002, 2003, 2006. The most common causes of completed suicide in boys under age 15 , was family problems while in girls was education failure, the most common reasons was economic factors for males in the 15-19 age group, while that of girls was family problems. The most common method of suicide in boys was hanging, while that of girls was firearm under 15 years of age, between the ages of 15-19 hanging for boys and the chemicals for girls was the most seen methods.

Conclusion It is essential to reduce the rate of suicide among child and adolescent so that prevention methods should be planned according to to age and gender outcomes of completed suicides.

\section{P405 CHILDREN WITH EATING DISORDERS PRESENTING TO NON-TERTIARY PAEDIATRIC UNITS IN IRELAND - A CASE SERIES}

${ }^{1}$ Aoife Branagan*, Evanne O'Halloran 1, ${ }^{2}$ Niofa Canty, 'Michael Brendan O'Neill, ${ }^{1}$ Hilary Stokes. ${ }^{1}$ Department of Paediatrics, Mayo University Hospital, Castlebar, Co Mayo, Ireland; ${ }^{2}$ Department of Paediatrics, Mayo University Hospital, Castlebar, Co Mayo, Ireland

\subsection{6/archdischild-2019-epa.751}

The number of young people with eating disorders is rapidly increasing and hospital admissions are rising. We describe a case series of seven females who required admission to a 